\title{
BMJ Open Preterm birth among Pacific Islander women and related perinatal outcomes: a scoping review protocol
}

\author{
Bohao Wu (D) , ${ }^{1}$ Kendall J Arslanian, ${ }^{2}$ Kate Nyhan, ${ }^{3,4}$ Sarah Taylor, ${ }^{5}$ \\ Veronika Shabanova, ${ }^{6,7}$ Bethel Muasau-Howard, ${ }^{8}$ Nicola L Hawley (D) ${ }^{1}$
}

To cite: Wu B, Arslanian KJ, Nyhan $\mathrm{K}$, et al. Preterm birth among Pacific Islander women and related perinatal outcomes: a scoping review protocol. BMJ Open 2021;11:e050483. doi:10.1136/ bmjopen-2021-050483

- Prepublication history for this paper is available online. To view these files, please visit the journal online (http://dx.doi. org/10.1136/bmjopen-2021050483).

Received 22 February 2021 Accepted 10 September 2021

\section{ABSTRACT}

Introduction Infants born alive $<37$ weeks are classified as premature. The global estimate of preterm birth in 2014 was $10.6 \%$, and it is the leading cause of death of children under the age of 5 years. Preterm birth disproportionately affects women of minority populations, yet knowledge about the incidence and associated outcomes among Pacific Islanders is limited. The objectives of this scoping review are to identify studies that describe risk factors, maternal-child health outcomes and existing interventions to prevent preterm birth among Pacific Islanders, and to summarise the barriers and facilitators to decrease the burden.

Methods and analysis We will follow the Joanna Briggs Institute Manual for Evidence Synthesis for scoping reviews and the Preferred Reporting Items for Scoping Reviews (PRISMA-ScR) to conduct this scoping review. The Covidence web application will be used for data management and consensus review. We will search on MEDLINE ALL (Ovid), EMBASE (Ovid), Web of Science Core Collection (as licensed at Yale), the Cochrane Library, CINAHL (EBSCOhost) and two non-indexed regional journals (Pacific Journal of Reproductive Health and Pacific Health Dialog). Title-abstract and full-text screening of eligible studies will be performed by two authors, and data will be extracted by the first author. Outcomes extracted will be presented using evidence mapping.

Ethics and dissemination Findings will drive suggestions for new data collection needed to fill knowledge gaps and improve future study designs to decrease the burden of preterm birth among Pacific Islanders. There are no ethical concerns. This protocol will be disseminated in related peer-reviewed journals.

\section{BACKGROUND}

According to the WHO, preterm birth is defined as babies born alive before 37 weeks of pregnancy are completed. ${ }^{1}$ Preterm birth is the leading cause of death globally in children under the age of 5 years $^{1}$ and is considered the most common cause of neonatal mortality (death in the first 28 days of life).$^{23}$ Common risk factors for preterm birth include maternal demographic characteristics, pregnancy history, nutritional status (both prepregnancy and interpregnancy),

\section{Strengths and limitations of this study}

To our knowledge, this will be the first scoping review to describe risk factors, perinatal outcomes and interventions related to preterm birth among Pacific Islanders.

- Findings from this review will guide future data collection to address gaps in existing literature to decrease the burden of preterm birth in this population.

- Quality assessment of the included studies will not be a primary emphasis of this review.

- Our future work will include a systematic review with meta-analyses based on our findings.

present pregnancy characteristics (eg, infectious conditions, hypertensive disorders and periodontal diseases), psychological characteristics (eg, stress and depression), smoking, infection, uterine contractions and cervical length. ${ }^{4-6}$ In the short term, compared with infants born full term (delivery between 37 week 0 day and 41 weeks 6 days $^{78}$ ), preterm infants are at increased risk of neonatal respiratory conditions, necrotising enterocolitis, sepsis, neuromotor abnormalities, and visual or hearing impairment. ${ }^{5910}$ Longer term consequences include issues related to neuromaturation (cognitive impairments, impaired learning ability and challenges with executive function ${ }^{9}$ ), and increased risk of lifelong morbidity, including hypertension, cardiometabolic disease, lung disfunction, etc. $^{11}$ Mothers who experience preterm birth have been shown to experience greater psychological distress compared with mothers who gave birth at full term. ${ }^{12}$ Furthermore, mothers who give birth before 37 weeks are at increased risk of having preterm infants in consequent pregnancies. ${ }^{4}$ Interventions to reduce morbidity and mortality associated with preterm birth can be classified as primary (implemented to all women before or during pregnancy), secondary (identifying women with known risk factors and reducing 
the risk) or tertiary (improving perinatal outcomes of preterm infants). ${ }^{13}$ Even with an increase in evidencebased interventions, the preterm birth rate globally has not declined, although survival among preterm infants has increased. ${ }^{13}$

The global prevalence estimate for preterm birth in 2014 was $10.6 \%$ (uncertainty interval $9.0 \%-12.0 \%$ ), ${ }^{14}$ and there are both between-country and within-country inequities. ${ }^{412} 13$ In the USA, for example, racial and ethnic differences in preterm birth rates were observed in a 2018 report from the Centers for Disease Control and Prevention; the preterm birth rate among nonHispanic black women (13.6\%) was almost 1.5 times the rate among non-Hispanic white women $(9.5 \%)$ in $2016 .{ }^{15}$ Little is currently known, however, about preterm birth among Pacific Islanders, who are the third fastest growing minority group in the USA-based on the 2010 US Census, 1.2 million people identified as native Hawaiian and other Pacific Islander. ${ }^{16}$ In the broader Pacific Ocean region, there are approximately 2.5 million people resident on over 12000 islands. ${ }^{17}$ Despite their population size, Pacific Islanders in the USA and the Pacific region in general have been historically under-represented in health research, likely related to limited healthcare access and a distrust of health professionals. ${ }^{18-21}$

The USA, New Zealand and Australia are countries with the largest Pacific Islander migrant populations in the world. In 2014, the preterm birth rate in the USA was 9.6\% (uncertainty interval not reported) and the number of preterm births in the USA placed it among the ten countries with the highest prevalence at that time,${ }^{14}$ while the rate in New Zealand was $7.5 \%$ (uncertainty interval $7.0 \%-$ 9.8\%), and in Australia 8.6\% (uncertainty interval 6.9\%$9.5 \%)$. Racial differences in preterm birth were observed in New Zealand and Australia: in New Zealand, Māori women had the highest rate of preterm birth compared with other Pacific women or European New Zealanders during the period 1980-2001. ${ }^{22}$ Similarly in Australia between 1984 and 2006, the prevalence of preterm birth among Aboriginal infants (14.8\%) was almost two times the prevalence among non-Aboriginal infants $(7.6 \%){ }^{23}$ In the USA and other countries or regions in the Pacific, our understanding of preterm birth is still limited.

Pacific Islanders have a unique health profile that may put them at risk for preterm or extremely preterm birth: they have a disproportionately high prevalence of obesity and related non-communicable diseases compared with other populations. ${ }^{17}$ Obesity is a significant risk factor for pre-eclampsia and prepregnancy diabetes, ${ }^{24}{ }^{25}$ which have been associated with indicated preterm births. ${ }^{4}$ In 13 Pacific Island nations described in the WHO STEPwise approach to surveillance (STEPS), over half of the population was overweight, and, notably, women had a greater burden of overweight and obesity compared with men. ${ }^{17}$ Similarly, high rates of obesity exists in the USA, where $38.7 \%$ of Pacific Islanders were obese, which was almost $9 \%$ higher than their white counterparts $(30.1 \%) .{ }^{26}$ According to the New Zealand Health Survey 2018/2019, the prevalence of obesity among New Zealanders aged over 15 years was $30.9 \%$; in adults, Maori were 1.8 times as likely to have obesity compared with non-Māori, and Pacific Islanders were 2.5 times as likely compared with non-Pacific adults. ${ }^{27}$ Likewise in Australia, obesity was the second highest contributor to disease in indigenous people. ${ }^{28}$

To better understand preterm birth and relevant perinatal outcomes among Pacific Islanders, the aim of this scoping review is to examine published original studies and reports about preterm birth among Pacific Islanders in the USA, the US Affiliated Pacific Islands (USAPIs) and three Pacific zones (Micronesia, Melanesia and Polynesia).

\section{OBJECTIVES}

The objective of this scoping review is to identify and summarise existing articles on preterm birth among Pacific Islander women. We will collect all published original studies that discuss preterm birth in the USA, the USAPIs and the wider Pacific region, including Micronesia, Melanesia and Polynesia. Because of the large Pacific diaspora present in Australia and New Zealand, we will also include studies from these settings. Specifically, we aim:

1. To summarise the data or reports about the prevalence of preterm birth among Pacific Islander women.

2. To identify risk factors for preterm birth among Pacific Islander women.

3. To understand short-term health consequences (diagnoses during the initial birth hospitalisation) and long-term health outcomes (symptoms or diagnoses after the initial birth hospitalisation) of preterm birth in infants born less than 37 gestational weeks.

4. To describe maternal health outcomes among Pacific Islander women who experienced preterm birth.

5. To explore whether there are interventions known to improve perinatal outcomes among Pacific Islander women and their preterm infants.

6. To identify whether the results of the above five objectives are different among Pacific Islanders in the USA, the USAPIs or the wider Pacific region.

7. To summarise knowledge gaps in the existing literature.

\section{METHODS}

The scoping review protocol will follow the Joanna Briggs Institute Manual for Evidence Synthesis for scoping reviews $^{29}$ and the Preferred Reporting Items for Scoping Reviews (PRISMA-ScR). ${ }^{30}$ The search strategy of this project has been posted on the Open Science Framework (OSF) ${ }^{31}$

\section{Eligibility criteria}

Studies will be included if they fulfil the following criteria 


\section{Study population}

Studies will be limited to Pacific Islanders living in the USA, the USAPIs, other countries or regions in three geographical zones in the Pacific, Micronesia, Melanesia and Polynesia. The list of included countries or regions follows the WHO definition of Pacific Island Countries ${ }^{32}$ and previous studies, ${ }^{1720}$ which includes American Samoa, Guam, Hawaii, the Commonwealth of the Northern Mariana Islands (CNMI), the Federated States of Micronesia (FSM), the Republic of the Marshall Islands (RMI), Palau, Kiribati, Nauru, Papua New Guinea, the Solomon Islands, Fiji, New Caledonia, Vanuatu, Tonga, Tuvalu, Tokelau, Niue, French Polynesia, New Zealand, Samoa and the Cook Islands. Moreover, since Australia has a large proportion of Pacific Islander residents (206673 people, $0.9 \%$ in 2016), ${ }^{33}$ studies from Australia will also be selected if they report outcomes among Pacific Islanders, including those from Ni-Vanuatu, Tahiti and the Pitcairn islands. In New Zealand and Hawaii, studies including Māori (the indigenous Polynesian people of New Zealand), the indigenous people of New Zealand, and Native Hawaiians of Hawaii will be selected.

\section{Outcomes of interest}

The main outcome of interest for this review will be preterm birth among Pacific Islanders. Prevalence of and risk factors associated with preterm birth in those studies will be summarised. Relevant health outcomes of preterm infants will be classified as 'short-term' (diagnoses during the initial birth hospitalisation) or 'long-term' (symptoms or diagnoses after the initial birth hospitalisation) outcomes. ${ }^{34} 35$ Health outcomes of mothers who experienced preterm birth will also be examined. Potential neonatal outcomes will include, but will not be limited to, birth weight, fetal growth restriction, fetal death, stillbirths, neonatal intensive care unit admission, neonatal and infant mortality, congenital abnormalities and longterm health effects. Maternal health outcomes included in our study will be, but will not be limited to, maternal mood effects, and physical health outcomes if there is any report. Existing interventions that have been implemented on preterm Pacific Islander infants will also be reviewed. Studies retrospectively examining changes in healthcare management or practices will not be included in this review; studies describing outcomes of multiple births will also be excluded.

\section{Publication date}

The search was completed on 5 November 2020, so we will include studies published prior to this date. No publication date limits were imposed in the databases; that is, each database was searched from inception.

\section{Publication type}

Original studies published in peer-reviewed journals and government reports will be included in this review. Dissertations will be eligible for inclusion. Conference abstracts and master's theses will not be included in the review since the final study outcomes may not be available/ reported, but these will be examined for the purpose of citation chaining. Case reports will also be excluded.

\section{Language}

Studies published in English will be included. If a study is written in a language other than English but the title and abstracts are in English, we will include the paper in the title abstract screening stage and attempt to obtain a translation of the full text should it be determined to be relevant to our outcomes of interest.

\section{Search strategy}

Literature search strategies will be developed using two concepts: (1) Pacific Islanders and (2) preterm birth outcomes. Appropriate controlled vocabulary terms and keyword search terms will be used. To the extent allowed by bibliographic database indexing, articles about NZ but only discussing NZ Europeans and articles about Australia but only including aboriginal Australians or EuropeanAustralians will not be retrieved.

The search strategy will be developed by author, BW, in consultation with all coauthors, including a medical librarian. The search histories for all databases will be archived on an OSF project ${ }^{31}$ in a reproducible format. Our search strategy on MEDLINE ALL (Ovid) is listed in table 1.

\section{Information sources}

We will search the following five databases: MEDLINE ALL (Ovid), EMBASE (Ovid), Web of Science Core Collection (as licensed at Yale, listed in box 1), the Cochrane Library, and CINAHL (EBSCOhost). Articles published in the Pacific Journal of Reproductive Health and Pacific Health Dialog, two regional journals, will be searched independently, since studies published in these two journals may be highly relevant, yet neither journal is well indexed in major bibliographic databases.

We will also conduct citation chaining (backwards and forwards) on papers that meet inclusion criteria and relevant reviews to identify additional studies that may have been missed in the initial search. Recent papers that cited included studies will also be reviewed, based on the citation graph in Web of Science Core Collection.

Reports from international, national, state and territorial government agencies will also be searched manually, including, but not limited to, WHO, the Centers for Disease Control, Pacific Island Health Officers Association, New Zealand Ministry of Health, Counties Manukau Health, and Australian Government Department of Health.

\section{Data management}

Search results will be downloaded from databases and imported to Covidence (an evidence synthesis web application) for deduplication, title-abstract screening and full-text screening. ${ }^{36}{ }^{37}$ Other relevant studies identified through hand-searching and citation chaining will also be added to the Covidence screening project. The 


\begin{tabular}{|c|c|c|}
\hline Number & Search terms & Results \\
\hline 1 & $\begin{array}{l}\text { exp premature birth/ or exp infant, premature/ or exp obstetric labor, premature/ or exp infant, low birth } \\
\text { weight/ or exp tocolytic agents/ or exp fetal membranes, premature rupture/ or exp infant, premature, } \\
\text { diseases/ or exp retinopathy of prematurity/ or exp respiratory distress syndrome, newborn/ or exp } \\
\text { kangaroo-mother care method/ or exp enterocolitis, necrotizing/ or exp bronchopulmonary dysplasia/ } \\
\text { or (delivery, obstetric/ and pregnancy outcome/) }\end{array}$ & 196985 \\
\hline 2 & 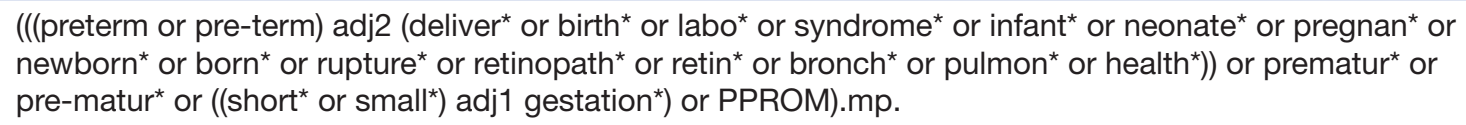 & 236244 \\
\hline 3 & 1 or 2 & 327774 \\
\hline 4 & oceania/ or Australasia/ or exp pacific islands/ or exp oceanic ancestry group/ & 70217 \\
\hline 5 & 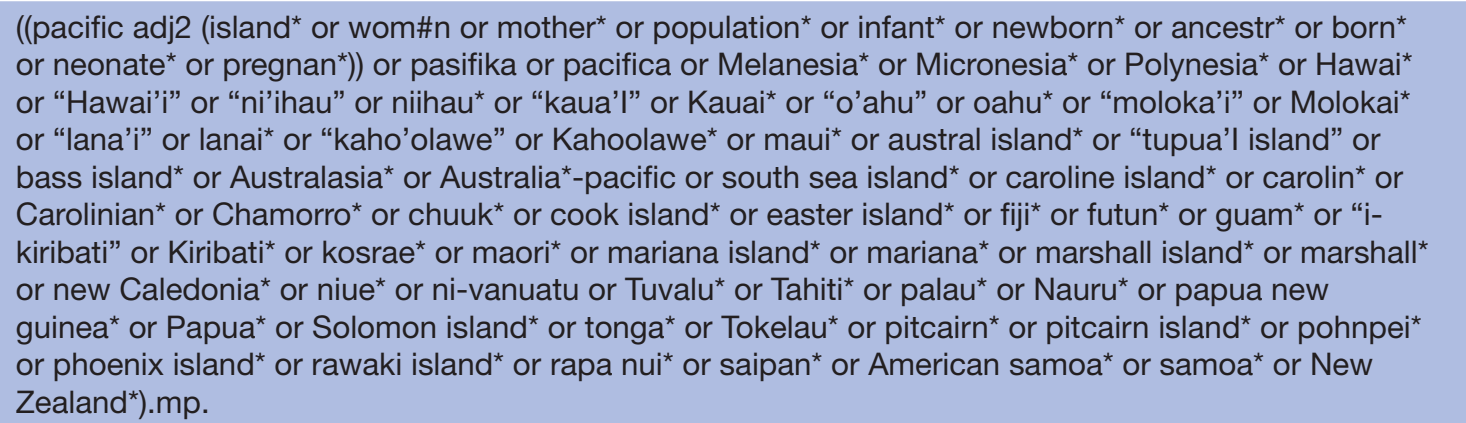 & 152302 \\
\hline 6 & 4 or 5 & 159981 \\
\hline 7 & 3 and 6 & 2251 \\
\hline 8 & exp animals/ not humans/ & 4752796 \\
\hline 9 & 7 not 8 & 2017 \\
\hline
\end{tabular}

study selection process will be reported in the PRISMA flowchart.

\section{Selection of sources and evidence}

We will pilot the inclusion and exclusion criteria before the main phase of title-abstract screening process. For the pilot, at least two authors will review 50 records, making title-abstract screening decisions (yes/no/maybe) and

\section{Box 1 Web of science core collection licensed at Yale}

Web of Science Core Collection: Citation Indexes (Yale's version)

- Science Citation Index Expanded (SCI-EXPANDED)-1900-present

- Social Sciences Citation Index (SSCI) - 1900-present

- Arts \& Humanities Citation Index (A\&HCI)-1975-present

- Conference Proceedings Citation Index-Science (CPCl-S) - 1991-present

- Conference Proceedings Citation Index-Social Science \& Humanities (CPCl-SSH)-1991-present

- Book Citation Index-Science (BKCl-S)-2005-present

- Book Citation Index-Social Sciences \& Humanities (BKCl-SSH)-2005-present

- Emerging Sources Citation Index (ESCI)-2015-present

Web of Science Core Collection: Chemical Indexes (Yale's version)

- Current Chemical Reactions (CCR-EXPANDED)-1985-present (Includes Institut National de la Propriete Industrielle structure data back to 1840)

Index Chemicus (IC) - 1993-present creating tags to be used in Covidence. Any disagreements will be discussed with the senior author to reach consensus on the interpretation of the inclusion and exclusion criteria. The piloted 50 records will be uploaded directly to the irrelevant or Full Text Screening category of the Covidence project.

Each article will be screened with two steps: (1) title and abstract screening; (2) full-text screening. Within each step, publications will be reviewed by two reviewers independently. Disagreements during the screening process on inclusion status will be discussed by the first and senior authors and consensus reached on their inclusion. The reason for exclusion will be recorded during the fulltext screening stage. Screening questions is expressed in box 2. We may record additional exclusion criteria as the review process proceeds.

\section{Data extraction process}

Articles that meet the eligibility criteria and pass the screening questions will be moved to the data extraction phase. The first author, BW, will extract relevant information from the articles and compare their extractions. A customised data-extraction sheet designed by the authors will be used to collect data and information that consist with the objectives of the scoping review (see box 3). We will pilot this extraction sheet on several studies to confirm all relevant information is being collected by this method. 


\section{Box 2 Screening questions}

1. Was the article or title-abstract published in the english language?

2. Does the article discuss preterm birth outcomes?

3. Are Pacific Islanders discussed in the article?

4. Does the article disaggregate preterm birth outcomes for Pacific Islanders rather than aggregating with other ethnic groups? (Exclude if aggregated)

5. Is the article a conference abstract or a master thesis? (Exclude if yes)

6. Is the article a case report? (Exclude if yes)

7. Is the article focused on outcomes after multiple birth? (Exclude if yes)

8. Does the article report preterm birth among women with specific medical condition only? (Exclude if yes)

Categories will be revised during the pilot extraction process if modifications are necessary. Extraction fields may change depending on the final included studies.

\section{Patient and public involvement}

Our study does not involve patients or the public in the design, conduct, reporting or dissemination plans.

\section{Study status}

Title-abstract and full-text screening are complete. Citation chaining and grey literature searching is in progress. We expect to complete the study by June 2022.

\section{Box 3 Data extraction categories}

Publication details

- Citation (year of publication and first author)

- Study type (original study/agency report/dissertation)

- Funding source

Study characteristics

- Study design/type

- Objective(s) of study

- Study location/setting (country of origin and data source)

Participant characteristics

- Maternal age/age range

- Pacific Islander subgroups

- Number of participants

- Socioeconomic status of participants

Characteristics of study setting

- Health system characteristics

- Economic development indicators

The Definition of Preterm Birth and Related Items

- The Upper and Lower Limit of Gestational Age of the Participants

- Gestational Age Measurement Method

Prevalence Estimates of Preterm Birth Reported

Risk Factors Reported

Relevant Short-term Infant Health Outcomes (diagnoses during the initial birth hospitalisation)

Relevant Long-term Infant Health Outcomes (symptoms or diagnoses after the initial birth hospitalisation)

Relevant Maternal Health Outcomes

Implemented Interventions and Outcomes

\section{PRESENTATION OF FINDINGS}

The information extracted from the selected articles will be presented by evidence mapping to express the breadth of research on preterm birth among Pacific Islanders. The data will be displayed according to year of publication, country of origin, study design, number of participants, Pacific Islander subgroups, prevalence estimates reported, risk factors reported, relevant infant and maternal postpartum outcomes reported, and existing interventions reported. Where intervention studies are identified, we will attempt to map their components to the WHO standards for improving quality of maternal and newborn care in health facilities to complement a recent, similar, review by Wilson $e t a l^{38}$ Data and figures will be summarised to express the outcomes. We will provide a narrative summary of the selected studies and discuss how our findings in this review relate to our objectives. If sufficient data are determined to be available to address one or more of our study objectives quantitatively, we will conduct meta-analyses in the future to summarise the findings.

\section{DISCUSSION}

The purpose of this scoping review is to understand knowledge gaps about preterm birth and relevant perinatal outcomes among Pacific Islander women and their infants. Our intention is that our review will guide future data collection to address gaps in the existing literature, identify risk factors associated with preterm birth among Pacific Islanders and assess the evidence for existing interventions to address poor perinatal outcomes associated with preterm birth in this group.

\section{ETHICS AND DISSEMINATION}

There are no apparent ethical issues. This study will be published in a peer-reviewed journal.

\section{Author affiliations}

${ }^{1}$ Department of Chronic Disease Epidemiology, Yale University School of Public Health, New Haven, Connecticut, USA

${ }^{2}$ Department of Social and Behavioral Sciences, Yale University School of Public Health, New Haven, Connecticut, USA

${ }^{3}$ Harvey Cushing/John Hay Whitney Medical Library, Yale University, New Haven, Connecticut, USA

${ }^{4}$ Department of Environmental Health Sciences, Yale University School of Public Health, New Haven, Connecticut, USA

${ }^{5}$ Division of Neonatal-Perinatal Medicine, Yale School of Medicine, New Haven, Connecticut, USA

${ }^{6}$ Department of Pediatrics, Yale School of Medicine, New Haven, Connecticut, USA ${ }^{7}$ Department of Biostatistics, Yale School of Medicine, New Haven, Connecticut, USA ${ }^{8}$ Department of Obstetrics and Gynecology, Lyndon B Johnson Tropical Medical Center, Pago Pago, American Samoa

Contributors BW and NLH conceived the study, with the support of ST, VS, KN and BM-H. BW, NLH and KN developed the search strategy. BW, KA and NLH wrote the initial draft of the manuscript, with the review of ST, VS, KN and BM-H. BW, KA and NLH will finish the study screening.

Funding This work was supported by the US National Institutes of Health (PI: Hawley, NLH, grant number R03HD093993) and China Scholarship Council (BW, 
grant number 201806010213). The funders had no role in the design, analysis or compiling this manuscript.

Competing interests None declared.

Patient and public involvement Patients and/or the public were not involved in the design, or conduct, or reporting, or dissemination plans of this research.

Patient consent for publication Not applicable.

Provenance and peer review Not commissioned; externally peer reviewed.

Open access This is an open access article distributed in accordance with the Creative Commons Attribution Non Commercial (CC BY-NC 4.0) license, which permits others to distribute, remix, adapt, build upon this work non-commercially, and license their derivative works on different terms, provided the original work is properly cited, appropriate credit is given, any changes made indicated, and the use is non-commercial. See: http://creativecommons.org/licenses/by-nc/4.0/.

\section{ORCID iDs}

Bohao Wu http://orcid.org/0000-0002-3278-196X

Nicola L Hawley http://orcid.org/0000-0002-2601-3454

\section{REFERENCES}

1 World Health Organization. Preterm birth, 2018. Available: https:// www.who.int/en/news-room/fact-sheets/detail/preterm-birth [Accessed Sep 2020].

2 Lawn JE, Kinney M. Preterm birth: now the leading cause of child death worldwide. Sci Trans/ Med 2014;6:263ed21.

3 McClure EM, Goldenberg RL, Jobe AH, et al. Reducing neonatal mortality associated with preterm birth: gaps in knowledge of the impact of antenatal corticosteroids on preterm birth outcomes in low-middle income countries. Reprod Health 2016;13:61.

4 Goldenberg RL, Culhane JF, lams JD, et al. Epidemiology and causes of preterm birth. Lancet 2008;371:75-84.

5 Vogel JP, Chawanpaiboon S, Moller A-B, et al. The global epidemiology of preterm birth. Best Pract Res Clin Obstet Gynaecol 2018;52:3-12.

6 Simmons LE, Rubens CE, Darmstadt GL, et al. Preventing preterm birth and neonatal mortality: exploring the epidemiology, causes, and interventions. Semin Perinatol 2010;34:408-15.

7 World Health Organization. Icd-10: international statistical classification of diseases and related health problems: tenth revision. 2nd ed. Switzerland, Geneva: World Health Organization, 2004. https://apps.who.int/iris/handle/10665/42980

8 Spong CY. Defining "term" pregnancy: recommendations from the Defining "Term" Pregnancy Workgroup. JAMA 2013;309:2445-6.

9 Allen MC, Cristofalo EA, Kim C. Outcomes of preterm infants: morbidity replaces mortality. Clin Perinatol 2011;38:441-54.

10 Kramer MS, Papageorghiou A, Culhane J, et al. Challenges in defining and classifying the preterm birth syndrome. Am J Obstet Gynecol 2012;206:108-12.

11 Luu TM, Rehman Mian MO, Nuyt AM. Long-Term impact of preterm birth: neurodevelopmental and physical health outcomes. Clin Perinatol 2017;44:305-14.

12 Yaari M, Treyvaud K, Lee KJ, et al. Preterm birth and maternal mental health: longitudinal trajectories and predictors. J Pediatr Psychol 2019;44:736-47.

13 lams JD, Romero R, Culhane JF, et al. Primary, secondary, and tertiary interventions to reduce the morbidity and mortality of preterm birth. Lancet 2008;371:164-75.

14 Chawanpaiboon S, Vogel JP, Moller A-B, et al. Global, regional, and national estimates of levels of preterm birth in 2014: a systematic review and modelling analysis. Lancet Glob Health 2019;7:e37-46.

15 Martin JA, Osterman MJK. Describing the increase in preterm births in the United States, 2014-2016. NCHS Data Brief 2018;312:1-8.

16 Lindsay Hixson BBH. Myoung Ouk Kim. the native Hawaiian and other Pacific Islander population: 2010: U.S. department of Commerce, economics and statistics administration, U.S. census bureau, 2012. Available: https://www.census.gov/prod/cen2010/ briefs/c2010br-12.pdf [Accessed Oct 2020].

17 Hawley NL, McGarvey ST. Obesity and diabetes in Pacific Islanders: the current burden and the need for urgent action. Curr Diab Rep 2015;15:29

18 Ayers BL, Hawley NL, Purvis RS, et al. Providers' perspectives of barriers experienced in maternal health care among Marshallese women. Women Birth 2018;31:e294-301.

19 George S, Duran N, Norris K. A systematic review of barriers and facilitators to minority research participation among African Americans, Latinos, Asian Americans, and Pacific Islanders. Am J Public Health 2014;104:e16-31.

20 McElfish PA, Ayers BL, Purvis RS, et al. Best practices for community-engaged participatory research with Pacific Islander communities in the USA and USAPI: protocol for a scoping review. BMJ Open 2018;8:e019653.

21 McElfish PA, Narcisse M-R, Long CR, et al. Leveraging communitybased participatory research capacity to recruit Pacific Islanders into a genetics study. J Community Genet 2017;8:283-91.

22 Mantell CD, Craig ED, Stewart AW, et al. Ethnicity and birth outcome: new Zealand trends 1980-2001: Part 2. pregnancy outcomes for Maori women. Aust N Z J Obstet Gynaecol 2004;44:537-40.

23 Langridge AT, Nassar N, Li J, et al. Social and racial inequalities in preterm births in Western Australia, 1984 to 2006. Paediatr Perinat Epidemiol 2010;24:352-62.

24 Doherty DA, Magann EF, Francis J, et al. Pre-pregnancy body mass index and pregnancy outcomes. Int $J$ Gynaecol Obstet 2006;95:242-7.

25 McDonald SD, Han Z, Mulla S, et al. Overweight and obesity in mothers and risk of preterm birth and low birth weight infants: systematic review and meta-analyses. BMJ 2010;341:c3428.

26 Centers for Disease Control and Prevention. Summary health statistics: National health interview survey, 2017. Available: https:// ftp.cdc.gov/pub/Health Statistics/NCHS/NHIS/SHS/2017 SHS Table_A-15.pdf [Accessed 17 Nov 2020]

27 New Zealand Ministry of Health. Annual update of key results 2018/19: new Zealand health survey, 2019. Available: https://www. health.govt.nz/publication/annual-update-key-results-2018-19-newzealand-health-survey [Accessed 17 Nov 2020].

28 National Preventative Health Taskforce. Australia: the healthiest country by 2020 a discussion paper. Available: http://healthwest.org. au/wp-content/uploads/2014/11/Australia-The-Healthiest-Countryby-2020.pdf [Accessed 17 Nov 2020].

29 Peters M, Godfrey C, Mclnerney P. The Joanna Briggs Institute reviewers' manual 2015: methodology for JBI scoping reviews, 2015. Available: https://wiki.jbi.global/display/MANUAL [Accessed Sep 2020].

30 Tricco AC, Lillie E, Zarin W, et al. PRISMA extension for scoping reviews (PRISMA-ScR): checklist and explanation. Ann Intern Med 2018;169:467-73.

31 et alWu B, Arslanian K, Nyhan K. Preterm birth among Pacific Islander women and related perinatal outcomes: a scoping review protocol, 2020. Available: https://osf.io/8x74v/ [Accessed Dec 2020].

32 World Health Organization. Pacific island countries, 2013. Available: https://apps.who.int/iris/bitstream/handle/10665/136831/ccsbrief_ pci en. pdf? sequence $=1 \&$ isAllowed $=y$ [Accessed Aug 2020]

33 Batley J. What does the 2016 census reveal about Pacific Islands communities in Australia? 2017. Available: https://devpolicy.org/ 2016-census-reveal-about-pacific-islands-communities-in-australia20170928/ [Accessed Nov 2020]

34 Chang $\mathrm{H}-\mathrm{Y}$, Sung $\mathrm{Y}-\mathrm{H}$, Wang S-M, et al. Short- and longterm outcomes in very low birth weight infants with admission hypothermia. PLoS One 2015;10:e0131976.

35 Patel RM. Short- and long-term outcomes for extremely preterm infants. Am J Perinatol 2016;33:318-28.

36 Kellermeyer L, Harnke B, Knight S. Covidence and Rayyan. Jmla 2018;106:580.

37 Babineau J. Product review: Covidence (systematic review software). J Can Health Libr Assoc 2014;35:68-71.

38 Wilson AN, Spotswood N, Hayman GS, et al. Improving the quality of maternal and newborn care in the Pacific region: a scoping review. Lancet Reg Health West Pac 2020;3:100028. 